\title{
CONFORMAL METRICS ON THE UNIT BALL: THE GEHRING-HAYMAN PROPERTY AND THE VOLUME GROWTH
}

\author{
TOMI NIEMINEN AND TIMO TOSSAVAINEN
}

\begin{abstract}
We continue the study of conformal metrics on the unit ball in Euclidean space. We assume that the density $\rho$ associated with the metric satisfies a Harnack inequality and then consider how much we can relax the volume growth condition from that in [Proc. London Math. Soc. Vol. 77 (3) (1998), 635-664] so that the Gehring-Hayman property still holds along the radii, i.e., if a boundary point can be accessed via a path with $\rho$-length $M<\infty$, then the $\rho$-length of the corresponding radius is bounded by $C M$. It turns out that if the path is inside a Stolz cone, then this result holds irrespective of the volume growth condition. Moreover, even if the path is not inside a Stolz cone, we are able to relax the volume growth condition for large $r$, and still conclude that the corresponding radius is $\rho$-rectifiable. This observation leads to a new estimate on the size of the boundary set corresponding to the $\rho$-unrectifiable radii.
\end{abstract}

\section{INTRODUCTION}

Given a continuous density $\rho: \mathbb{B}^{n} \rightarrow \mathbb{R}_{+}$, we define a conformal metric $d_{\rho}$ by setting

$$
\operatorname{length}_{\rho}(\gamma)=\int_{\gamma} \rho(z)|d z|
$$

for a curve $\gamma$ in $\mathbb{B}^{n}$, and

$$
d_{\rho}(x, y)=\inf _{\gamma} \operatorname{length}_{\rho}(\gamma) \text { for } x, y \in \mathbb{B}^{n},
$$

where the infimum is taken over all curves joining $x$ and $y$ in $\mathbb{B}^{n}$. We also define a measure $\mu_{\rho}$ by setting

$$
\mu_{\rho}(E)=\int_{E} \rho^{n} d m_{n} \quad \text { for a Borel set } E \subset \mathbb{B}^{n},
$$

where $m_{n}$ denotes the $n$-dimensional Lebesgue measure.

We assume that the density $\rho$ satisfies a Harnack inequality, i.e., there exists a constant $A \geq 1$ so that

$$
\frac{1}{A} \leq \frac{\rho(x)}{\rho(y)} \leq A
$$

Received by the editors June 28, 2009.

2010 Mathematics Subject Classification. Primary 30C65.

Key words and phrases. Boundary, conformal metrics, Gehring-Hayman property, quasiconformal mapping .

(C)2009 American Mathematical Society Reverts to public domain 28 years from publication 
whenever $x, y \in B\left(z, \frac{1}{2}(1-|z|)\right)$ for some $z \in \mathbb{B}^{n}$. We also assume a growth condition on the isodiametric profile of $\left(\mathbb{B}^{n}, d_{\rho}\right)$ which we, following [2], define as a function $\eta_{\rho}:\left[0, \operatorname{diam}_{\rho}\left(\mathbb{B}^{n}\right)\right] \rightarrow[0, \infty]$,

$$
\eta_{\rho}(r)=\sup \left\{\mu_{\rho}(D): D \subset \mathbb{B}^{n} \text { and } \operatorname{diam}_{\rho}(D) \leq r\right\} .
$$

Notice that the condition $\eta_{\rho}(r) \leq B r^{n}$ for all $r>0$ is equivalent to assuming the so-called volume growth condition [1],

$$
\mu_{\rho}\left(B_{\rho}(x, r)\right) \leq B r^{n} \quad \text { for all } x \in \mathbb{B}^{n} \text { and } r>0
$$

The motivation for conformal metrics arises primarily from the theory of quasiconformal mappings. Recall that the average derivative

$$
a_{f}(x)=\left(\frac{1}{m_{n}\left(B_{x}\right)} \int_{B_{x}} J_{f} d m_{n}\right)^{1 / n}, \quad B_{x}=B\left(x, \frac{1}{2}(1-|x|)\right),
$$

of a quasiconformal mapping $f: \mathbb{B}^{n} \rightarrow \Omega$ is a primary example of a density satisfying the above conditions. However, not all conformal densities arise from a quasiconformal mapping; see [1] for more information and examples.

The main question in this paper is whether it is possible to relax the condition (1.1) so that the Gehring-Hayman property still holds along radii, i.e., if it is possible to join $\xi \in \partial \mathbb{B}^{n}$ and 0 by a path $\tilde{\gamma}$ with $\operatorname{length}_{\rho}(\tilde{\gamma})=M<\infty$, then $\operatorname{length}_{\rho}([0, \xi)) \leq$ $C M$ where $C \geq 1$ is a finite constant. In [1, Theorem 3.1] it is namely shown that assuming a Harnack inequality and (1.1) guarantees that the geodesic arc in $\mathbb{B}^{n}$ essentially is the shortest path with respect to $\rho$-distance between any $x$ and $y$ in $\overline{\mathbb{B}}^{n}$. Nevertheless, the authors of [1] do not comment in any way whether (1.1) is the best possible upper bound for the volume growth or not.

It appears that the answer to our question depends fundamentally on whether we allow $\tilde{\gamma}$ to be an arbitrary path in the unit ball or do we restrict it, for example, in a cone-shape neighborhood of $\xi$. Indeed, for a Stolz cone at $\xi \in \partial \mathbb{B}^{n}$,

$$
\text { Cone }(\xi, \lambda, h)=\bigcup\{B(t \xi, \lambda(1-t)): 1-h \leq t<1\},
$$

where $\lambda \in(0,1)$ and $h \in(0,1]$, we have the following theorem.

Theorem 1.1. Let $\tilde{\gamma} \subset \operatorname{Cone}(\xi, \lambda, 1)$ be a curve joining 0 and $\xi$ so that length $\rho(\tilde{\gamma})=$ $M<\infty$. Then length $\rho([0, \xi)) \leq C M$ where $C=C(A, \lambda, n) \geq 1$, i.e., $C$ is a finite constant depending only on $A, \lambda$ and $n$ and nothing else.

In other words, Theorem 1.1 holds independently of the volume growth. On the other hand, for arbitrary paths joining $\xi$ and 0 in the unit ball, the situation is quite different. The only known relevant method, which has been used, e.g., in [1], is based on estimating the modulus of the families of paths and, as evaluation of examples like $\eta_{\rho}(r)=B r^{n}(\log (1+1 / r))^{p}, p>0$, reveals, it does not allow us to relax exceedingly the volume growth condition for small $r$. However, if we replace (1.1) with the condition

$$
\eta_{\rho}(r) \leq B r^{n} \beta(r) \quad \text { for all } r>0
$$

where $\beta$ is a positive increasing function on $(0, \infty)$ with $\lim _{r \rightarrow 0} \beta(r)=1$, we have the following theorem.

Theorem 1.2. Let $\tilde{\gamma} \subset \mathbb{B}^{n}$ be a curve joining 0 and $\xi$ so that length $(\tilde{\gamma})=M<\infty$. If the density $\rho$ satisfies $(1.2)$, then length ${ }_{\rho}([0, \xi)) \leq C M$ where $C=C(A, B, n, \xi)$ $\geq 1$. 
Since the constant $C$ in Theorem 1.2 depends also on $\xi$, we cannot conclude the Gehring-Hayman theorem under volume growth (1.2) in general; nevertheless, we have the following corollary.

Corollary 1.3. Let $\tilde{\gamma} \subset \mathbb{B}^{n}$ be a curve joining 0 and $\xi$ so that length $(\tilde{\gamma})<\infty$. If the density $\rho$ satisfies $(1.2)$, then also length ${ }_{\rho}([0, \xi))<\infty$.

Our final theorem is an extension of the Radial Limit Theorem [1, Theorem 4.4]. This result also relates to [3, Remark 1.3] where the size of the boundary set $E \subset \partial \mathbb{B}^{n}$ where the conformal deformation mapping can "blow up" was estimated.

Theorem 1.4. Let $E=\left\{\xi \in \partial \mathbb{B}^{n}:\right.$ length $\left._{\rho}([0, \xi))=\infty\right\}$. If $\rho$ satisfies

$$
\eta_{\rho}(r) \leq B r^{n}(\log (e+r))^{p}
$$

for some $B>0$ and $0 \leq p<n-1$, then $\operatorname{cap}_{n}(E)=0$.

Here, $\operatorname{cap}_{n}$ denotes the conformal $n$-capacity. Recall that $\operatorname{cap}_{n}(E)=0$ for a Borel set $E$ if and only if

$$
\inf \left\{\int_{\mathbb{R}^{n}}|\nabla u|^{n} d m_{n}: u \in C^{\infty}\left(\mathbb{R}^{n}\right), u \mid K \geq 1 \text { and } u \mid\left(\mathbb{R}^{n} \backslash B(0,2) \equiv 0\right)\right\}=0
$$

for all compact sets $K \subset E$.

As a final remark we note that our Theorem 1.1 is an analogue of the inequality (4.1) in 1] (whose proof can be found, e.g., in [4, Lemma 1.3]); the difference is that the inequality (4.1) gives an upper bound for the $\rho$-diameter of the Stolz cone at $\xi$ in terms of the $\rho$-diameter of the arc $[(1-h) \xi, \xi)$. This inequality plays an important role, for example, in proving many results of [1] and 4].

\section{Proofs of the Results}

Proof of Theorem 1.1. Fix any $\lambda \in(1 / 2,1)$. It is easy to check that if the density $\rho$ satisfies the Harnack inequality with the constant $A \geq 1$ for all balls $B\left(x, \frac{1}{2}(1-|x|)\right)$, then it satisfies the inequality with another constant $A^{\prime}=A^{\prime}(A, \lambda, n) \geq A$ for all balls $B(x, \lambda(1-|x|))$, too.

Let $\xi \in \partial \mathbb{B}^{n}$ be so that there is a curve $\tilde{\gamma} \subset \operatorname{Cone}(\xi, \lambda, 1)$ with endpoints 0 and $\xi$ so that $\operatorname{length}_{\rho}(\tilde{\gamma})=M<\infty$. Denote by $\gamma$ the radius $[0, \xi)$.

For $\tilde{\gamma}_{j}$, a closed subcurve of $\tilde{\gamma}$ which connects the two boundary components of the annulus

$$
A_{j}=\bar{B}\left(\xi, 2^{-j}\right) \backslash B\left(\xi, 2^{-j-1}\right)
$$

in $A_{j} \cap \operatorname{Cone}(\xi, \lambda, 1)$, and for $\gamma_{j}=\gamma \cap A_{j}$, it holds that

$$
\operatorname{length}\left(\tilde{\gamma}_{j}\right) \geq \operatorname{length}\left(\gamma_{j}\right)=2^{-j-1} \text {. }
$$

Since every point of $A_{j} \cap \operatorname{Cone}(\xi, \lambda, 1)$ can be joined to $\gamma_{j}$ with a finite number (depending only on $n$ ) of balls of type $B(x, \lambda(1-|x|)$ ), the Harnack inequality implies that there is a constant $C=C\left(A^{\prime}\right)$ so that

$$
C \operatorname{length}_{\rho}\left(\tilde{\gamma}_{j}\right) \geq \operatorname{length}_{\rho}\left(\gamma_{j}\right) \text {. }
$$

The claim follows now from summing over $j$ :

$$
\operatorname{length}_{\rho}(\gamma)=\sum_{j} \operatorname{length}_{\rho}\left(\gamma_{j}\right) \leq \sum_{j} C \operatorname{length}_{\rho}\left(\tilde{\gamma}_{j}\right) \leq C \operatorname{length}_{\rho}(\tilde{\gamma})=C M
$$


The proof of our main result, Theorem 1.2, is similar in spirit to the proof of the classical Gehring-Hayman theorem in [1]. Let us first recall that the modulus $\bmod \Gamma \in[0, \infty]$ of a family $\Gamma$ of curves in $\mathbb{B}^{n}$ is defined as

$$
\bmod \Gamma=\inf _{\tilde{\rho}} \int_{\mathbb{B}^{n}} \tilde{\rho}^{n} d m_{n} .
$$

Here the infimum is taken over all Borel measurable densities $\tilde{\rho}: \mathbb{B}^{n} \rightarrow[0, \infty]$ which are admissible. A density $\tilde{\rho}$ is admissible, if $\operatorname{length}_{\tilde{\rho}}(\gamma) \geq 1$ for all $\gamma \in \Gamma$.

If $E$ and $F$ are subsets of $\mathbb{B}^{n}$, then we denote by $\bmod \left(E, F ; \mathbb{B}^{n}\right)$ the modulus of the family of all rectifiable curves in $\mathbb{B}^{n}$ connecting $E$ and $F$. Let us recall a well-known lower bound for $\bmod \left(E, F ; \mathbb{B}^{n}\right)$ : If $E$ and $F$ are disjoint continua in $\mathbb{B}^{n}$, then

$$
\phi_{n}(t) \leq \bmod \left(E, F ; \mathbb{B}^{n}\right)
$$

where

$$
t=\frac{\operatorname{dist}(E, F)}{\min \{\operatorname{diam}(E), \operatorname{diam}(F)\}}
$$

and

$$
\phi_{n}(t)=\frac{\omega_{n-1}}{2\left[\log \left(\lambda_{n}(1+t)\right)\right]^{n-1}} .
$$

Here $\omega_{n-1}$ is the surface area of $\partial \mathbb{B}^{n}$ and $\lambda_{n} \geq 1$ is a constant that depends only on $n$.

Let us also recall the fact that $\bmod \left(E, F ; \mathbb{B}^{n}\right)=\infty$ whenever $E$ and $F$ are connected non-degenerate sets in $\overline{\mathbb{B}^{n}}$ whose closures have non-empty intersection. See, for example, [5] for the proof of these elementary properties of the modulus.

Our next lemma extends [1, Lemma 3.2].

Lemma 2.1. Suppose that $\rho$ satisfies (1.2) and let $E$ be a non-empty subset of $\mathbb{B}^{n}$ and let $L \geq \delta>0$. Assume that $\operatorname{diam}_{\rho}(E) \leq \delta$ and that $\Gamma$ is a family of curves in $\mathbb{B}^{n}$ so that every curve $\gamma \in \Gamma$ has one endpoint in $E$ and length ${ }_{\rho}(\gamma) \geq L$. Then

$$
\bmod \Gamma \leq \frac{C \beta(4 L)}{[\log (1+L / \delta)]^{n-1}}
$$

where $C=C(B, n) \geq 1$.

Proof. Define $\tilde{\rho}: \mathbb{B} \rightarrow[0, \infty)$ by

$$
\tilde{\rho}(x)= \begin{cases}\frac{\rho(x)}{(\log (1+L / \delta))\left(\delta+\operatorname{dist}_{\rho}(x, E)\right)} & \text { for } x \in \mathbb{B}^{n}, \operatorname{dist}_{\rho}(x, E)<L, \\ 0 & \text { elsewhere. }\end{cases}
$$

This function is clearly Borel measurable and we claim that $\tilde{\rho}$ is an admissible density for the curve family $\Gamma$.

Let $\gamma \in \Gamma$. We may assume that $\gamma: I \rightarrow \mathbb{B}^{n}$ has an arc-length parametrization with $I=[0, \operatorname{length}(\gamma)]$ and $\gamma(0) \in E$. For $s \in I$, let $t(s)$ be the $\rho$-length of $\gamma \mid[0, s]$, i.e.,

$$
t(s)=\int_{0}^{s} \rho(\gamma(u)) d u
$$

Obviously, $\operatorname{dist}_{\rho}(\gamma(s), E) \leq t(s)$ for all $s \in I$. Moreover, since $t(s)$ is a continuous increasing function of $s$ and $t(\operatorname{length}(\gamma))=\operatorname{length}_{\rho}(\gamma) \geq L$, there exists a smallest 
number $s_{0} \in I$ with $t\left(s_{0}\right)=L$. It now follows from the definition of $\tilde{\rho}$ that

$$
\begin{aligned}
\operatorname{length}_{\rho}(\gamma) & \geq \frac{1}{\log (1+L / \delta)} \int_{0}^{s_{0}} \frac{\rho(\gamma(s))}{\delta+\operatorname{dist}_{\rho}(\gamma(s), E)} d s \\
& \geq \frac{1}{\log (1+L / \delta)} \int_{0}^{L} \frac{d t}{\delta+t}=1 .
\end{aligned}
$$

Thus $\tilde{\rho}$ is admissible.

Select a point $x_{0} \in E$ and let $k$ be the smallest positive integer with $\delta+L \leq 2^{k+1} \delta$. Since $\delta \leq L$, taking logarithms gives

$$
1 \leq k \leq \frac{\log (1+L / \delta)}{\log 2}
$$

Since $\operatorname{diam}_{\rho}(E) \leq \delta$, we have that $\left\{x \in \mathbb{B}^{n}: \operatorname{dist}_{\rho}(x, E)<L\right\} \subset B_{\rho}\left(x_{0}, 2^{k+1} \delta\right)$.

Let $B_{j}=B_{\rho}\left(x_{0}, 2^{j} \delta\right)$ for $j \in \mathbb{N}$. Then $\tilde{\rho}$ vanishes outside $B_{k+1}$ and so

$$
\bmod \Gamma \leq \int_{\mathbb{B}^{n}} \tilde{\rho}^{n} d m_{n}=\int_{B_{k+1}} \tilde{\rho}^{n} d m_{n}=\int_{B_{1}} \tilde{\rho}^{n} d m_{n}+\sum_{j=1}^{k} \int_{B_{j+1} \backslash B_{j}} \tilde{\rho}^{n} d m_{n} .
$$

Since $\operatorname{dist}_{\rho}(x, E) \geq 2^{j} \delta-\delta$ for $x \in B_{j+1} \backslash B_{j}$ and $j \in \mathbb{N}$, we obtain

$$
\begin{aligned}
\bmod \Gamma & \leq \frac{1}{[\log (1+L / \delta)]^{n}}\left(\int_{B_{1}} \frac{\rho^{n}}{\delta^{n}} d m_{n}+\sum_{j=1}^{k} \int_{B_{j+1} \backslash B_{j}} \frac{\rho^{n}}{2^{j n} \delta^{n}} d m_{n}\right) \\
& \leq \frac{1}{[\log (1+L / \delta)]^{n}}\left(\frac{\mu_{\rho}\left(B_{1}\right)}{\delta^{n}}+\sum_{j=1}^{k} \frac{1}{2^{j n} \delta^{n}} \mu_{\rho}\left(B_{j+1}\right)\right) .
\end{aligned}
$$

The conditions (1.2) and (2.2) and the definition of $k$ now imply that

$$
\begin{aligned}
\bmod \Gamma & \leq \frac{1}{[\log (1+L / \delta)]^{n}}\left(2^{n} B \beta(2 \delta)+\sum_{j=1}^{k} 2^{n} B \beta\left(2^{j+1} \delta\right)\right) \\
& =\frac{2^{n} B(k+1) \beta(4 L)}{[\log (1+L / \delta)]^{n}} \\
& \leq \frac{2^{n} B / \log 2}{[\log (1+L / \delta)]^{n-1}} \beta(4 L) .
\end{aligned}
$$

Lemma 2.1 is proven.

Proof of Theorem 1.2. Let $\xi \in \partial \mathbb{B}^{n}$ be so that there is a curve $\tilde{\gamma} \subset \mathbb{B}^{n}$ with endpoints 0 and $\xi$ so that length $\rho(\tilde{\gamma})=M<\infty$. Denote by $\gamma$ the radius $[0, \xi)$.

Again, for each $j \in \mathbb{N}$, consider the annulus

$$
A_{j}=\bar{B}\left(\xi, 2^{-j}\right) \backslash B\left(\xi, 2^{-j-1}\right)
$$

and let $\tilde{\gamma}_{j}$ be a closed subcurve of $\tilde{\gamma}$ which connects the two boundary components of $A_{j}$ in $A_{j} \cap \mathbb{B}^{n}$ and $\gamma_{j}=\gamma \cap A_{j}$.

Observe first that

$$
\bmod \left(\gamma_{j}, \tilde{\gamma}_{j} ; \mathbb{B}^{n}\right) \geq c_{1}(n)>0 .
$$

This observation follows simply from the estimate $(2.1)$ and that $\operatorname{dist}\left(\gamma_{j}, \tilde{\gamma}_{j}\right) \leq$ $2^{-j} \leq 2 \min \left\{\operatorname{diam}\left(\gamma_{j}\right), \operatorname{diam}\left(\tilde{\gamma}_{j}\right)\right\}$

On the other hand, if length $\rho(\alpha) \geq c_{2} \operatorname{length}_{\rho}\left(\tilde{\gamma}_{j}\right)$ for all curves $\alpha$ that join $\gamma_{j}$ and $\tilde{\gamma}_{j}$ in $\mathbb{B}^{n}$, then also length $\rho(\alpha) \geq c_{2} \operatorname{diam}_{\rho}\left(\tilde{\gamma}_{j}\right)$, and Lemma 2.1 and the facts 
that length $\rho(\tilde{\gamma})$ is finite and $\lim _{r \rightarrow 0} \beta(r)=1$, and therefore $\beta(r) \leq 10$ for small $r>0$, hence imply that there is a finite $j_{0}$ (depending on $\xi$ ) so that

$$
\bmod \left(\gamma_{j}, \tilde{\gamma}_{j} ; \mathbb{B}^{n}\right) \leq \frac{C^{\prime} \beta\left(4 c_{2} \text { length }_{\rho}\left(\tilde{\gamma}_{j}\right)\right)}{\left[\log \left(1+c_{2}\right)\right]^{n-1}} \leq \frac{10 C^{\prime}}{\left[\log \left(1+c_{2}\right)\right]^{n-1}}
$$

for all $j \geq j_{0}$. Here $C^{\prime}$ is the constant in Lemma 2.1. But by the lower bound estimate (2.3) this is impossible if the constant $c_{2}$ is sufficiently large depending on $B$ and $n$. Hence we deduce that for each $j \geq j_{0}$ there is a curve $\alpha_{j}$ connecting the sets $\gamma_{j}$ and $\tilde{\gamma}_{j}$ so that

$$
\operatorname{length}_{\rho}\left(\alpha_{j}\right) \leq c_{2} \operatorname{length}_{\rho}\left(\tilde{\gamma}_{j}\right)
$$

where $c_{2}=c_{2}(B, n)$.

Let us next consider two cases according to whether the euclidean length of $\alpha_{j}$ is or is not substantially smaller than the euclidean length of $\gamma_{j}$.

Suppose first that length $\left(\alpha_{j}\right) \leq \frac{1}{10} \operatorname{length}\left(\gamma_{j}\right)$. Since $\alpha_{j}$ connects $\gamma_{j}$ and $\tilde{\gamma}_{j}$, we have that $\operatorname{dist}\left(\gamma_{j}, \tilde{\gamma}_{j}\right) \leq \frac{1}{10} \operatorname{length}\left(\gamma_{j}\right)$. However, we also have length $\left(\gamma_{j}\right) \leq$ $\operatorname{length}\left(\tilde{\gamma}_{j}\right)$ which means that $\tilde{\gamma}_{j}$ must have a subcurve of euclidean length comparable to length $\left(\gamma_{j}\right)$ near the Whitney ball containing $\gamma_{j}$. It now follows from the Harnack inequality that length ${ }_{\rho}\left(\gamma_{j}\right) \leq c_{3} \operatorname{length}_{\rho}\left(\tilde{\gamma}_{j}\right)$ for some $c_{3}$ depending only on $A$.

On the other hand, if length $\left(\alpha_{j}\right)>\frac{1}{10} \operatorname{length}\left(\gamma_{j}\right)$, then it again follows from the Harnack inequality that length ${ }_{\rho}\left(\gamma_{j}\right) \leq c_{4}$ length $_{\rho}\left(\alpha_{j}\right)$ for some $c_{4}$ depending only on $A$. By combining this with $(2.5)$ we arrive at length ${ }_{\rho}\left(\gamma_{j}\right) \leq c_{2} c_{4} \operatorname{length}_{\rho}\left(\tilde{\gamma}_{j}\right)$. We conclude that for all $j \geq j_{0}$ we have

$$
\operatorname{length}_{\rho}\left(\gamma_{j}\right) \leq c_{5} \operatorname{length}_{\rho}\left(\tilde{\gamma}_{j}\right)
$$

with a constant $c_{5}=c_{2} c_{4}$ depending on $A, B$ and $n$.

Finally, we have to deal also with the subcurves $\gamma_{j}$ and $\tilde{\gamma}_{j}$ for $j<j_{0}$. Since 0 is in the closure of both $\gamma$ and $\tilde{\gamma}$, and length $\left(\gamma_{j}\right) \leq \operatorname{length}\left(\tilde{\gamma}_{j}\right)$ for all $j$, it follows from the Harnack inequality that there is a constant $c_{6}$ depending only on $A, n$, and $j_{0}$ so that

$$
\operatorname{length}_{\rho}\left(\bigcup_{j<j_{0}} \gamma_{j}\right) \leq c_{6} \operatorname{length}_{\rho}\left(\bigcup_{j<j_{0}} \tilde{\gamma}_{j}\right) \text {. }
$$

Since $j_{0}$ depends only on $\xi$, it follows that there is a constant $C=\max \left\{c_{5}, c_{6}\right\}$ depending only on $A, B, n$ and $\xi$ so that

$$
\operatorname{length}_{\rho}(\gamma)=\sum_{j} \operatorname{length}_{\rho}\left(\gamma_{j}\right) \leq \sum_{j} C \operatorname{length}_{\rho}\left(\tilde{\gamma}_{j}\right) \leq C \operatorname{length}_{\rho}(\tilde{\gamma}) .
$$

Theorem 1.2 now follows.

Proof of Theorem 1.4. First we have to show that $E$ is a Borel set. For $k \in \mathbb{N}$, let $E_{k}=\left\{\xi \in \partial \mathbb{B}^{n}: \operatorname{length}_{\rho}([0, \xi)) \leq k\right\}$. Let $\left(\xi_{i}\right)$ be a sequence in some $E_{k}$ with $\left(\xi_{i}\right) \rightarrow \xi$. Then, for any $\lambda \in[0,1)$, we have that

$$
\operatorname{length}_{\rho}([0, \lambda \xi])=\int_{0}^{\lambda} \rho(t \xi) d t=\lim _{i \rightarrow \infty} \int_{0}^{\lambda} \rho\left(t \xi_{i}\right) d t \leq k .
$$

This implies that

$$
\operatorname{length}_{\rho}([0, \xi))=\lim _{i \rightarrow \infty} \operatorname{length}_{\rho}([0, \lambda \xi)) \leq k
$$


and so $\xi \in E_{k}$. It follows that $E_{k}$ is closed and therefore the set $E=\partial \mathbb{B}^{n} \backslash$ $\left(\bigcup_{k \in \mathbb{N}} E_{k}\right)$ is a Borel set.

Let $F=\bar{B}\left(0, \frac{1}{2}\right)$ and consider the family $\Gamma$ of curves in $\mathbb{B}^{n}$ connecting $E$ and $F$. Assume towards a contradiction that $\operatorname{cap}_{n}(E)>0$. Then also $\bmod \Gamma>0$. On the other hand, Lemma 2.1 provides us an upper bound

$$
\bmod \Gamma \leq \frac{C(\log (e+4 L))^{p}}{[\log (1+L / \delta)]^{n-1}} .
$$

Now, if length $\rho(\alpha)=\infty$ for all $\alpha \in \Gamma$, then we may choose $\delta=1$ and $L$ arbitrarily large in Lemma 2.1, which implies $\bmod \Gamma=0$ which is a contradiction. Therefore there exists a curve $\alpha \in \Gamma$ with length ${ }_{\rho}(\alpha)<\infty$.

Let $\xi \in E$ and $x \in F$ be the endpoints of $\alpha$. Since $x$ can be connected to 0 by a curve of finite $\rho$-length, there exists a curve $\tilde{\gamma}$ in $\mathbb{B}^{n}$ with endpoints 0 and $\xi$ and $\operatorname{length}_{\rho}(\tilde{\gamma})<\infty$. It follows from Corollary 1.3 that $\operatorname{length}_{\rho}([0, \xi))<\infty$ which is a contradiction with the definition of $E$.

\section{REFERENCES}

1. M. Bonk, P. Koskela and S. Rohde, Conformal metrics on the unit ball in euclidean space, Proc. London Math. Soc. Vol 77 (3) (1998), 635-664. MR1643421 (99f:30033)

2. B. Hanson, P. Koskela and M. Troyanov, Boundary behavior of the quasi-regular maps and the isodiametric profile, Conform. Geom. Dyn. Vol 5 (2001), 81-99. MR1872158 (2002h:30025)

3. T. Nieminen and T. Tossavainen, Boundary behavior of conformal deformations, Conform. Geom. Dyn. Vol 11 (2007), 56-64. MR2314242 (2008c:30031)

4. T. Tossavainen, On the connectivity properties of the $\rho$-boundary of the unit ball, Ann. Acad. Sci. Fenn. Math. Diss. 123, 2000. MR1763839 (2002b:30024)

5. J. Väisälä, Lectures on n-dimensional quasiconformal mappings. Lecture Notes in Mathematics 120, Springer, New York, 1989.

Department of Technology, Jyväskylä University of Applied Sciences, P.O. Box 207, FIN-40101 JYVÄSKYLÄ, FinLAND

E-mail address: tomi.nieminen@jamk.fi

Department of Teacher Education, University of Joensud, P.O. Box 86, Fin-57101 Savonlinna, Finland

E-mail address: timo.tossavainen@joensuu.fi 